JÓZEF WIKTOROWICZ

Warszawa

\title{
DIE GRAMMATIKALISIERUNG DER PARTIKEL SCHON
}

Unter der Grammatikalisierung versteht man in der Regel die Entwicklung einer lexikalischen Einheit zu einem grammatischen Morphem bzw. zu einem grammatischen Funktionswort. Meist wird der Begriff der Grammatikalisierung im Zusammenhang mit der Entwicklung von lexikalischen Einheiten zu grammatischen Morphemen verwendet, aber in den letzten Jahren wird der Begriff der Grammatikalisierung auch auf die Entwicklung von Autosemantika zu Synsemantika verstanden. Bei Kuryłowicz tauchte der Begriff der Grammatikalisierung noch im engeren Sinne auf, denn Kuryłowicz hat die Grammatikalisierung in dem Sinne aufgefasst, dass die lexikalischen Morpheme zu grammatischen Morphemen werden. ${ }^{1}$ Heutzutage aber wird zu den Grammatikalisierungsprozessen auch die Entwicklung von lexikalischen Einheiten zu anderen lexikalischen Einheiten, die eine stark eingeschränkte lexikalische Bedeutung aufweisen. Die Entstehung von Grad- und Abtönungspartikeln aus Adverbien bzw. aus Konjunktionen wird ebenfalls zur Grammatikalisierung gerechnet. ${ }^{2}$ Die Ausdehnung des Grammatikalisierungsbegriffs auf die Entwicklung von Adverbien bzw. Konjunktionen zu Grad- und Abtönungspartikeln ist berechtigt, denn auch bei solchen Grammatikalisierungsprozessen durchlaufen die lexikalischen Einheiten bestimmte Phasen der grammatischen Entwicklung. Im Laufe der Sprachentwicklung zeigen manche lexikalische Einheiten einen allmählichen Schwund an semantischen Merkmalen, sie verlieren auch ihre freien Wortstellungsmöglichkeiten zugunsten einer festen Stellung im Satz. Damit verlieren bestimmte lexikalische Einheiten ihren Status eines freien Morphems zugunsten eines Status des gebundenen Morphems.

Im Folgenden soll gezeigt werden, wie sich das frühneuhochdeutsche Adverb schon ('schön') zur Gradpartikel schon entwickelt hat.

\footnotetext{
${ }^{1}$ Kuryłowicz (1987: 140): „Gra matykalizacja polega na wzroście zakresu użycia jakiegoś wykładnika formalnego (formantu), który z leksykalnego awansuje na gramatyczny lub z mniej gramatycznego na bardziej gramatyczny (np. z przyrostka słowotwórczego na końcówkę fleksyjną".

${ }^{2}$ Vgl. Z.B. Autenrieth (2005).
} 
Noch in der mittelhochdeutschen Periode haben sich viele Adverbien von den verwandten Adjektiven durch den Mangel des Umlauts voneinander unterschieden, z.B. herte - harte, senfte - sanfte, veste - faste, spcete - spâte, schoene - schône usw. (Paul 1982: § 141). In der frühneuhochdeutschen Zeit kommt es häufig zum Schwund einer lexikalischen Form bzw. entwickeln sich die beiden lexikalischen Formen auseinander und der lexikalische Zusammenhang zwischen den beiden Formen geht damit verloren. Solch eine semantische Auseinanderentwicklung ist unter anderem bei den etymologisch verwandten Wortformen schön - schon und fest - fast eingetreten.

In der Anfangsphase des Frühneuhochdeutschen wurde das Adverb schon ('schön') sehr oft in Kombination mit Verben verwendet, die eine Handlung oder einen Vorgang bezeichnen. Damit blieb der semantische Zusammenhang mit dem Adjektiv schön noch erhalten.

Als sy nun gen hoff kamen, ward Fortunatus von dem künig schon entpfangen.

[Anonym: Fortunatus. Deutsche Literatur von Luther bis Tucholsky, S. 598

(vgl. Anonym-Fortunatus, S. 85)]

Also wurden vil töchtern schon geklait, die sunst noch lang müßten on so gůte klayder gewesen sein. [Anonym: Fortunatus. Deutsche Literatur von Luther bis Tucholsky, S. 563 (vgl. Anonym-Fortunatus, S. 64)]

Di Meola (2000): Die Grammatikalisierung deutscher Präpositionen.

erwachet die künigin vnd legt sich schon an.

[Anonym: Fortunatus. Deutsche Literatur von Luther bis Tucholsky, S. 671

(vgl. Anonym-Fortunatus, S. 128)]

Das Adverb schon konnte auch durch eine Partikel näher bestimmt werden: gar schon, so schon, nit so schon:

$\mathrm{Zu}$ denen kam Fortunatus vnd empfiengen ainander gar schon in frembden landen [Anonym: Fortunatus. Deutsche Literatur von Luther bis Tucholsky, S. 485 (vgl. Anonym-Fortunatus, S. 17)]

sy dankt ir gar züchtigklichen vnd naigt sich so schon, das die äbtissin wol sach, das sy von edlem stammen geboren was,

[Anonym: Fortunatus. Deutsche Literatur von Luther bis Tucholsky, S. 679

(vgl. Anonym-Fortunatus, S. 133)]

do ward er nit so schon gegrüßt vnd enpfangen, als andre mal,

[Anonym: Fortunatus. Deutsche Literatur von Luther bis Tucholsky, S. 498

(vgl. Anonym-Fortunatus, S. 25)]

Neben der Verwendung als Adverb schon (in der Bedeutung 'schön') taucht im Frühneuhochdeutschen relativ früh das Wort schon in Kontexten auf, in denen das Wort schon als Gradpartikel interpretiert werden muss. In vielen Kontexten erscheint 
schon nicht mehr in der Bedeutung eines modalen Adverbs, das eine Handlung oder einen Vorgang charakterisiert, sondern als Gradpartikel. Damit beobachten wir den Prozess der semantischen Abnutzung des Adverbs schon und das Wort schon entwickelt sich zum Vertreter einer anderen syntaktischen Klasse.

Als Gradpartikel erscheint schon in frühneuhochdeutschen Texten vor einem Bezugswort oder vor einer Wortgruppe und kennzeichnet eine Erwartungshaltung des Sprechers. Wenn das Bezugswort eine Zeitangabe ist, drückt schon aus, dass der betreffende Sachverhalt früher als erwartet vor dem genannten Zeitpunkt eingetreten ist:

den Berg: ohne das eine nit/welches sie nur halb hinauff zogen/dieweil es schon zuvor halber hinab gelauffen gewesen:

[Anonym: Das Lalebuch. Deutsche Literatur von Luther bis Tucholsky, S. 1737 (vgl. Anonym-Lalebuch, S. 38)]

da kam des Schweynhirten Tochter/bey deren er schon zuvor ein Nacht gewesen/an jhn/griff jhn mit herben scharpffen worten an/schalte jn wie ein todtes Roß [Anonym: Das Lalebuch. Deutsche Literatur von Luther bis Tucholsky, S. 1838 (vgl. Anonym-Lalebuch, S. 111)]

und ich erfordere von euch keine ehrerbietung einer unterthanin/sondern die liebe einer Königin/zu der ich euch bestimmet/und die ihr schon fürlängst in meinem herzen gewesen seit. [Anton Ulrich Herzog von Braunschweig: Die durchleuchtige Syrerin Aramena. Deutsche Literatur von Luther bis Tucholsky, S. 14789

(vgl. Anton-Aramena, S. 41)]

Die Gradpartikel schon kann auch vor verschiedenen Mengenangaben stehen, und sie drückt dann aus, dass eine bestimmte Menge (in Bezug auf Entfernung, Zeit, Größe) früher als erwartet erreicht wurde:

Dieser Assyrische Prinz ware/vor unserer ankunft/schon etliche monden daselbst gewesen. [Anton Ulrich Herzog von Braunschweig: Die durchleuchtige Syrerin Aramena. Deutsche Literatur von Luther bis Tucholsky, S. 14813

(vgl. Anton-Aramena, S. 60)]

Nachdem sie diß gespräche noch etwas erlängert/und die nacht schon zimlich weit eingebrochen war/verliesse sie ihn/

[Anton Ulrich Herzog von Braunschweig: Die durchleuchtige Syrerin Aramena. Deutsche Literatur von Luther bis Tucholsky, S. 15066

(vgl. Anton-Aramena, S. 262)]

Darum/(antwortete ich/) daß ich alsdann versichert wäre/dermaleins durch eure hÔlfe in der Dianen tempel nach Ninive zu kommen / von der man mich schon zweimal unbilliger weise entfÔret hat. [Anton Ulrich Herzog von Braunschweig: Die durchleuchtige Syrerin Aramena. Deutsche Literatur von Luther bis Tucholsky, S. 15194 (vgl. Anton-Aramena, S. 365)] 
Als ich nachmals zu dem Orgas wieder kame/ware der Prinzessin flucht schon allenthalben kund worden

[Anton Ulrich Herzog von Braunschweig: Die durchleuchtige Syrerin Aramena. Deutsche Literatur von Luther bis Tucholsky, S. 15634

(vgl. Anton-Aramena, S. 56)]

Das Wort schon erscheint weiterhin in Kombination mit Verben; es drückt aber nicht mehr die modale Bedeutung aus, sondern nur die Erwartung des Sprechers, dass ein Sachverhalt früher als erwartet eingetreten ist. In solchen Fällen können wir noch von einer adverbialen Verwendung sprechen, aber das Adverb schon verliert viel von seinem semantischen Gehalt, denn es drückt die Erwartung des Sprechers aus, und nicht mehr unter welchen Umständen ein Sachverhalt eingetreten ist. Damit ist das Wort schon nicht mehr erfragbar:

Also stehet ietzund diese sache/und haben wir hoch vonnöten/alles wol in ordnung zu stellen/weil in sechs tagen die hochzeit schon fortgehen soll.

[Anton Ulrich Herzog von Braunschweig: Die durchleuchtige Syrerin Aramena. Deutsche Literatur von Luther bis Tucholsky, S. 16673

(vgl. Anton-Aramena, S. 175)]

Ingerman/der damals die Mirina von Basan schon liebte/wurde höchst erfreut

[Anton Ulrich Herzog von Braunschweig: Die durchleuchtige Syrerin Aramena. Deutsche Literatur von Luther bis Tucholsky, S. 16767

(vgl. Anton-Aramena, S. 251)]

Er fande daselbst die wägen und pferde schon färtig

[Anton Ulrich Herzog von Braunschweig: Die durchleuchtige Syrerin Aramena. Deutsche Literatur von Luther bis Tucholsky, S. 16351

(vgl. Anton-Aramena, S. 668)]

Dieser erzehlte uns das anbringen des gesandten/welches wir ohnedas schon errahten hatten [Anton Ulrich Herzog von Braunschweig: Die durchleuchtige Syrerin Aramena. Deutsche Literatur von Luther bis Tucholsky, S. 15961

(vgl. Anton-Aramena, S. 334)]

Und hat schon dazumahl ein sichere Propheceyung geschienen/weil diser Heil. Orden von sieben Florentiner herstammet/da $\beta$ er auch absonderlich in der Catholischen Kirchen floriren werde.

[Abraham a Sancta Clara: Mercks Wienn. Deutsche Literatur von Luther bis Tucholsky, S. 1952 (vgl. Abraham-Wien, S. 78)]

Im Hinblick auf das Kriterium der Erfragbarkeit könnte man das Wort schon als Partikel betrachten, andererseits ist dieses Wort im Hinblick auf Wortstellungsmöglichkeiten noch nicht auf eine feste Position eingeschränkt. Wenn wir andere Beispiele mit dem Wort schon betrachten, so können wir sehen, dass schon innerhalb des Satzes seine Position verändern kann: 
kam dieselbe mit der Aramena/und bemühete sich/dieser bekümmerten Fürstin gemüt müglichst zu frieden zu sprechen: vorbringend/wie daß sie schon nachrichtung von Eliesers bässerung hätte.

[Anton Ulrich Herzog von Braunschweig: Die durchleuchtige Syrerin Aramena. Deutsche Literatur von Luther bis Tucholsky, S. 14803

(vgl. Anton-Aramena, S. 52)]

Weil ich schon in freundes land war/auch von des reichsstatthalters in Elam kammerherren begleitet wurde;

[Anton Ulrich Herzog von Braunschweig: Die durchleuchtige Syrerin Aramena. Deutsche Literatur von Luther bis Tucholsky, S. 15107

(vgl. Anton-Aramena, S. 294)]

Die ankunft des Esau ware diesem KÑnig höchst angenem/weil dessen dapferkeit ihme schon bekant war:

[Anton Ulrich Herzog von Braunschweig: Die durchleuchtige Syrerin Aramena. Deutsche Literatur von Luther bis Tucholsky, S. 15457

(vgl. Anton-Aramena, S. 574)]

Weil Esau schon aus dem gerüchte bekant war / als thÉte man ihm grosse ehre an: [Anton Ulrich Herzog von Braunschweig: Die durchleuchtige Syrerin Aramena. Deutsche Literatur von Luther bis Tucholsky, S. 15507

(vgl. Anton-Aramena, S. 614)]

Für die Erststellung von schon konnten im Frühneuhochdeutschen keine Belege gefunden werden, aber im Neuhochdeutschen gibt es relativ viele Belege für die Anfangsstellung von schon:

Schon ist er kein Verschwender mehr,

Schon giebt er Schmeichlern kein Gehör.

[Gellert: Fabeln und Erzählungen. Deutsche Literatur von Luther bis Tucholsky, S. 155474 (vgl. Gellert-W Bd. 1, S. 199)]

Schon leuchtet der Morgen; die Morgenluft weht;

Schon wirbelt die Schwalbe den Morgengesang,

[Bürger: Gedichte (Ausgabe 1789). Deutsche Literatur von Luther bis Tucholsky, S. 81732 (vgl. Bürger-G Bd. 1, S. 161)]

Schon war es Abend geworden, und kalt und feucht wehte die herbstliche Luft. [Ahlefeld: Marie Müller. Deutsche Literatur von Luther bis Tucholsky, S. 7049 (vgl. Ahlefeld-Marie, S. 187)]

Neben der häufigen Verwendung von schon als Gradpartikel lassen sich im Frühneuhochdeutschen auch einige Beispiele für den Gebrauch von schon als Abtönungspartikel finden. Die Verwendung von schon als Abtönungspartikel scheint erst am Anfang zu stehen, aber es gibt Kontexte, in denen der Sprecher die Hoffnung 
signalisiert, dass der von ihm genannte Sachverhalt eintreten wird, obwohl es Hindernisse gibt, die die Realisierung des betreffenden Sachverhalts erschweren könnten. Die Verwendung von schon als Abtönungspartikel lässt sich in Aussagesätzen feststellen, in denen von einem zukünftigen Sachverhalt die Rede ist:

Tiribaces wird sich schon trösten lassen/wann er die hofnung/sie zu bekommen/ einmal verlieret. [Anton Ulrich Herzog von Braunschweig: Die durchleuchtige Syrerin Aramena. Deutsche Literatur von Luther bis Tucholsky, S. 16130

(vgl. Anton-Aramena, S. 479)]

/so verhoffeten doch die Assyrier / nun sie nur diesen ihren Prinzen wieder hatten/ dessen entwerdung sie so sehr beängstigt/es wÔrde mit ihm schon auch wieder gut werden. [Anton Ulrich Herzog von Braunschweig: Die durchleuchtige Syrerin Aramena. Deutsche Literatur von Luther bis Tucholsky, S. 16502

(vgl. Anton-Aramena, S. 40)]

Simede thut euch unrecht / und ich werde sie schon zur gesunden vernunft zu bringen wissen/daß sie anderst mit euch ümgehe.

[Anton Ulrich Herzog von Braunschweig: Die durchleuchtige Syrerin Aramena. Deutsche Literatur von Luther bis Tucholsky, S. 16518

(vgl. Anton-Aramena, S. 52)]

Dieser Aramenes wird schon mit dem bloßen leben und geistlichen stand müßen zufrieden seyn [Anton Ulrich Herzog von Braunschweig: Die durchleuchtige Syrerin Aramena. Deutsche Literatur von Luther bis Tucholsky, S. 16981 (vgl. Anton-Aramena, S. 421)]

Wenn wir das lexikalische Vorkommen des Wortes schon in den Texten im Laufe der frühneuhochdeutschen Periode zusammenfassen, so können wir sehen, dass das Wort schon im 15. und 16. Jahrhundert noch ausschließlich als modales Adverb in der Bedeutung schön verwendet wurde, während im 17. Jahrhundert die Verwendung von schon als Gradpartikel stark zunimmt und die Verwendung von schon als Adverb (in der Bedeutung 'schön') stark abnimmt. Im 18. Jahrhundert (d.h. schon in der neuhochdeutschen Periode) ist die adverbiale Verwendung von schon völlig verschwunden. Das Wort schon wird vorwiegend als Gradpartikel gebraucht und allmählich setzt sich auch der Gebrauch von schon als Abtönungspartikel durch. Damit ist der Übergang des Wortes schon von der Wortklasse Adverb in die Wortklasse Partikel vollzogen. Das Wort schon weist damit solche Merkmale wie Nichterfragbarkeit, Nichtverschiebbarkeit, Weglassbarkeit ohne grammatische Inkorrektheit eines Satzes hervorzurufen (vgl.u.a. Darski 2010: 111).

\section{Quellen}

Anonym: Fortunatus Vermutlich von einem Augsburger Bürger 1490 verfasst. Erstdruck: Augsburg (Johann Otmar) 1509. [Anonym: Deutsche Literatur von Luther bis Tucholsky, S. 460]. 
Anonym: Das Lalebuch. Erstdruck: Straßburg (Bernhard Jobins Erben) 1597.

[Anonym: Deutsche Literatur von Luther bis Tucholsky, S. 1691].

Abraham a Sancta Clara: Mercks Wienn. Erstdruck: Wien (Vivian) 1680.

[Abraham a Sancta Clara: Deutsche Literatur von Luther bis Tucholsky, S. 1894].

Charlotte von Ahlefeld: Marie Müller.

Erstdruck: Berlin (Johann Friedrich Unger) 1799.

[Ahlefeld: Deutsche Literatur von Luther bis Tucholsky, S. 6891].

Anton Ulrich Herzog von Braunschweig: Die durchleuchtige Syrerin Aramena. Erstdruck: Nürnberg (Hofmann) 1669-1673.

[Anton Ulrich Herzog von Braunschweig: Deutsche Literatur von Luther bis Tucholsky, S. 14714].

Gottfried August Bürger: Gedichte (Ausgabe 1789) Ausgabe letzter Hand: Göttingen (Dieterich) 1789.

[Bürger: Deutsche Literatur von Luther bis Tucholsky, S. 81419].

Christian Fürchtegott Gellert: Fabeln und Erzählungen.

Erstdrucke der Sammlung: [Erstes Buch:] Leipzig (Wendler) 1746, [Zweites Buch:] Leipzig (Wendler) 1748, [Drittes Buch, in:] Lehrgedichte und Erzählungen, Leipzig (Wendler) 1754.

[Gellert: Deutsche Literatur von Luther bis Tucholsky, S. 155133].

Digitale Bibliothek Bd. 125. Deutsche Literatur von Luther bis Tucholsky.

\section{Literatur}

Autenrieth, T. (2005): Grammatikalisierung bei Modalpartikeln. Das Beispiel eben. In: Grammatikalisierung im Deutschen. Hrsg. von T. Leuschner, T. Mortelmans, S. De Groodt. Berlin-New York: de Gruyter, S. 309-334.

Darski, J. (2010): Deutsche Grammatik. Ein völlig neuer Ansatz. Frankfurt am Main-Berlin: Peter Lang (Posener Beiträge zur Germanistik, Bd. 26).

Di Meola, C. (2000): Die Grammatiklaisierung deutscher Präpositionen. Tübingen: Stauffenburg.

Helbig, G., Buscha, J. (1998): Deutsche Grammatik. Ein Handbuch für den Ausländerunterricht. Leipzig-Berlin: Langenscheid, Verlag Enzyklopädie.

Kuryłowicz, J. (1987): Studia językoznawcze. Wybór prac opublikowanych w języku polskim. Warszawa: PWN.

Paul, H. (1982): Mittelhochdeutsche Grammatik. 22. durchgesehene Auflage von H. Moser, I. Schöbler, S. Grosse. Tübingen: Niemeyer.

Szczepaniak, R. (2009): Grammatikalisierung im Deutschen. Ein Einführung. Tübingen: Narr. 